\title{
Medicina veterinária e a saúde única: ação socioeducativa aplicada a idosos no contexto da COVID-19
}

\author{
Ana Laura Martins Ferreira ${ }^{1}$, Raquelline Figueiredo Braz $^{2}$, Mariana Comassio Chueiri ${ }^{1}$, Deborah Araujo \\ Policarpo ${ }^{1}$, Andrezza Brigato Siqueiraa ${ }^{1}$, Denise Ramos Pacheco ${ }^{1}$, Roberta Torres de Melo $^{3}$
}

Resumo: A saúde única integra as esferas da saúde humana, ambiental e animal, por meio de políticas públicas focadas na prevenção e controle de enfermidades infecciosas. O médico veterinário é um profissional atuante na área da saúde pública pelo contato direto com as três esferas, e uma das principais ações desse profissional inclui a promoção de medidas comunitárias educativas para disseminar informações em relação à prevenção de doenças zoonóticas. Diante da atual pandemia, desenvolvemos o projeto: "Efeito borboleta aplicado a asilos de UberlândiaMG no combate à COVID-19" com o objetivo de divulgar informações sobre a doença de maneira lúdica, e atender as demandas das instituições envolvidas. Foram produzidos e distribuídos dez materiais didáticos como vídeos e jogos interativos com orientações em relação à COVID-19 em 16 abrigos de idosos, junto à arrecadação de produtos de limpeza e higiene pessoal, máscaras e gêneros alimentícios, para enfatizar as medidas preventivas, provenientes de doações de 23 empresas. Funcionários das instituições desenvolveram as atividades propostas com os idosos, ressaltando a importância do distanciamento social e da higiene pessoal no momento atual. A ação executada permitiu a ampliação do conhecimento a respeito da pandemia, em virtude do uso de metodologias aplicadas às dificuldades e necessidades dos idosos, aliada à motivação emocional utilizada na proposta, o que favoreceu o processo ensino-aprendizagem. A presente ação social nas instituições auxiliou na conscientização e esclarecimento de dúvidas em relação à COVID-19 ao principal grupo de risco, e reforçou o contexto de atuação do médico veterinário na saúde única.

Palavras-chave: Educação; Epidemia; Grupo vulnerável; Promoção de saúde

\section{Veterinary medicine and the one health system: socio-educational action applied to elderly in the context of COVID-19}

Abstract: The one health system integrates the human, environmental, and animal health spheres through public policies focused on preventing and controlling infectious diseases. The veterinary doctor is a professional actor in public health through direct contact with the three spheres. One of these professional's main actions includes promoting community educational measures to disseminate information regarding the prevention of zoonotic diseases. In the face of the current pandemic, we developed the project: "Butterfly effect applied to asylums of Uberlândia, Minas Gerais State, Brazil, in the combat to COVID-19" to spread information about the disease playfully and to attend to the demands of the involved institutions. Ten educational materials were produced and distributed, such as videos and interactive games with guidelines regarding COVID19 , in 16 shelters for the elderly, along with the collection of cleaning and personal hygiene products, masks, and food products from donations from 23 companies, to emphasize preventive measures. Institutional staff developed the proposed activities with the elderly, emphasizing the importance of social distance and personal hygiene at this time. The action performed allowed the amplification of knowledge about the pandemic due to the use of methodologies applied to the difficulties and needs of the elderly, allied to the emotional motivation used in the proposal, which favored the teaching-learning process. This social action in the institutions helped to raise awareness and clarify doubts concerning VOCID-19 to the leading risk group and reinforced the context of the veterinarian's action in the one health.

Keywords: Education; Epidemic; Vulnerable group; Health promotion
Originais recebidos em

11 de junho de 2020

Aceito para publicação em

$1^{\circ}$ de dezembro de 2020

1

Acadêmica do curso de Medicina Veterinária na Universidade Federal de Uberlândia, Minas Gerais, Brasil.

2 Mestranda do Programa de Pós-Graduação em Ciências Veterinárias (PPGCV) na Universidade Federal de Uberlândia, Minas Gerais, Brasil 3 Docente de Saúde Pública do curso de Medicina Veterinária e do PPGCV na Universidade Federal de Uberlândia, Minas Gerais, Brasil

(autora para correspondência) roberta.tmelo@ufu.br 


\section{Introdução}

O conceito de saúde única foi elencado pela primeira vez por Rudolf Virchow (1821-1902), médico e político alemão do século XIX, que defendia a necessidade de haver um equilíbrio entre a saúde humana, animal e ambiental (Brown, 2003). Apesar disso, a ideia só ganhou espaço a partir de 2008, momento em que as organizações mundiais da Saúde (OMS), da Saúde Animal (OIE) e das Nações Unidas para alimentação e agricultura (FAO) instituíram os objetivos da saúde única na promoção de políticas públicas voltadas à prevenção e controle de enfermidades infecciosas (King et al., 2008).

Junto ao conceito de saúde única, Virchow também trouxe a denominação de zoonoses, compreendida pelas enfermidades infecciosas transmissíveis entre humanos e animais. Hoje está bem estabelecido que $60 \%$ das doenças infecciosas que acometem humanos são de origem animal e a globalização, aliada às mudanças climáticas e aos hábitos peculiares de diferentes sociedades, tem levado à emergência de zoonoses, como a COVID-19 (Chen et al., 2020).

A formação dos médicos veterinários é aliada a conteúdos que envolvem não só a área biomédica animal, mas também aspectos sociais voltados à atenção primária, ligada à prevenção de enfermidades zoonóticas e à promoção da saúde humana. Essa atuação ganhou ainda mais espaço com a inclusão do profissional aos Núcleos de Apoio à Saúde da Família (NASF), passando a atuar junto aos demais profissionais de saúde na avaliação de fatores de risco ligados à interação homem-animal, na prevenção e controle de zoonoses, em ações socioeducativas na comunidade, e na vigilância em saúde (Ministério da Saúde, Portaria n. 2488, de 21 de outubro de 2011).

A recente pandemia da COVID-19, iniciada na província de Wuhan, China, tornou ainda mais clara a influência das interações homem-animal silvestre na emergência de doenças zoonóticas. Trata-se de uma enfermidade cujo agente causador é um coronavírus (SARS-CoV-2). As pesquisas até então publicadas sugerem que o vírus se mantém de forma comensal em algumas espécies de morcegos e se adaptou aos pangolins, espécie que apresenta amplo contato com humanos nos mercados chineses (Zhang et al., 2020).

A América Latina recebeu o status de epicentro da pandemia a partir de maio de 2020, sendo o Brasil o país mais atingido, com mais de 1,600 milhão infectados e mais de 64 mil mortos, e até o momento o município de Uberlândia contabiliza mais de 4.324 casos confirmados, dos quais $486(11,2 \%)$ são idosos (Ministério da Saúde, 2020a). A doença se configura pelo seu status de maior risco em pacientes idosos, principalmente aqueles com comorbidades. As dificuldades inerentes a esse grupo de risco impossibilitam uma melhor compreensão da situação vivenciada, em virtude da ocorrência comum de alterações cognitivas, que inviabilizam a compreensão e adaptação às orientações de controle, mesmo aquelas mais simples (Alzheimer Europe, 2020).

As instituições de longa permanência para idosos apresentam carências não só relacionadas aos cuidados com esse grupo de risco, mas também deficiências estruturais e de recursos humanos, que permitiriam uma melhor forma de controle de doenças como a COVID-19. A sinergia desses fatores é refletida no primeiro grande estudo da COVID-19 em um lar de idosos, em que quase dois terços dos residentes foram infectados por um período de três semanas, com uma taxa de mortalidade de 33\% (McMichael et al., 2020). Apesar de ser uma questão alarmante, ainda é muito baixo o reconhecimento dado aos desafios únicos a serem enfrentados nessas instituições.

A Organização Mundial da Saúde definiu que procedimentos de isolamento e formas de educação em saúde adaptadas aos residentes de lares de idosos devem ser excepcionalmente elaboradas, considerando o comprometimento cognitivo significativo de parte deles. O número de pessoas dessa classe social que se 
sentem solitárias e deprimidas já é muito alto em condições normais, com $30 \%$ dos residentes de asilos com depressão em sua fase aguda. Com a inclusão das medidas de isolamento e distanciamento social esse valor pode alcançar de 50-55\% (Read et al., 2020).

A comunicação aberta, adequada, compreensiva, repetida e gradual a respeito da importância da doença é fundamental para favorecer a sensibilização ao conceito de prevenção contra a COVID-19. Além disso, precauções adicionais que abordem conselhos práticos de forma lúdica sobre as restrições ao contato social e medidas intensivas de higiene pessoal foram recomendadas, uma vez que podem auxiliar não só no combate à pandemia, mas também na redução dos impactos negativos na saúde mental decorrentes das restrições de convívio com familiares (World Health Organization [WHO], 2020).

Diante desse contexto, a atuação do profissional médico veterinário se torna fundamental no quesito orientação e conscientização da comunidade geral, principalmente aos idosos. Isso subsidiou o objetivo do presente trabalho de desenvolver e aplicar materiais educativos com conteúdo informativo lúdico voltado às necessidades do principal grupo de risco envolvido na COVID-19, e reforçar a informação por meio da entrega de doações de produtos relacionados às medidas de combate ao SARS-CoV-2.

\section{Procedimentos metodológicos}

As atividades extensionistas do projeto "Efeito Borboleta aplicado a asilos de Uberlândia no combate à COVID19" foram executadas pelos alunos coordenadores do Grupo de Estudos sobre Zoonoses (GEZ), da Faculdade de Medicina Veterinária (FAMEV), da Universidade Federal de Uberlândia (UFU). A proposta foi desenvolvida no período de março a junho de 2020.

Para obter domínio teórico sobre o novo coronavírus (SARS-CoV-2), os alunos envolvidos participaram de uma palestra apresentada pela orientadora do grupo, na qual foram apresentadas informações a respeito da definição, etiologia, histórico, epidemiologia, sintomas, diagnóstico, tratamento e controle da doença.

\section{Determinação das propostas didáticas, instituições e doações}

Para as ações educativas a respeito da atual pandemia da COVID-19 foram desenvolvidas dez propostas didáticas que abordaram aspectos relacionados às características, origem, relação com animais, sintomas, transmissão e, principalmente, às formas de combate ao novo coronavírus. Adicionalmente, foi disponibilizada uma planilha com telefones e endereços de todos os postos de saúde da cidade de Uberlândia para facilitar o acesso dos asilos às informações, à medida que surgirem atualizações sobre a COVID-19. A elaboração dos jogos incluiu estratégias lúdicas com o objetivo de informar e conscientizar os idosos sobre o coronavírus, de maneira adaptada às necessidades desse grupo. De forma complementar, os materiais abordaram aspectos ligados à manutenção da saúde mental por meio do uso de mensagens positivas e estimuladoras, em meio à pandemia.

A escolha das instituições beneficiadas foi baseada em uma pesquisa em 35 asilos da cidade de Uberlândia, MG. Desses, 16 foram selecionados pelo aspecto filantrópico, pela rápida resposta e retorno formal à realização de todas as atividades do projeto, em virtude da dificuldade de maiores contatos em época de isolamento social. Os abrigos contemplam um total de 364 idosos, que apresentam diferentes níveis de capacidade cognitiva e morbidades crônicas.

Com o objetivo de intensificar de forma ainda mais prática a proposta, foi inserida uma ação de cunho social, ligado à arrecadação de produtos de higiene pessoal e limpeza, assim como máscaras descartáveis e de dupla camada de algodão $100 \%$, e gêneros alimentícios para todos os residentes das instituições (disponível em 
Material Suplementar 1). As doações foram provenientes de um total de 23 empresas.

\section{Material didático físico}

Todo material físico foi produzido de acordo com a quantidade específica de residentes de cada asilo. No total foram elaborados quatro jogos, dois materiais informativos e uma simulação de coronavírus produzido em biscuit.

Os jogos interativos incluem o 'Jogo da Memória: conhecendo os sintomas da COVID-19', o jogo 'Como Controlar o Coronavírus', a 'Cruzadinha da COVID-19' e o 'Bingo da COVID-19'. Os materiais informativos foram a planilha de contatos das Unidades Básicas de Saúde de Uberlândia-MG e o folder 'Coronavírus e a COVID19: Você sabia?'.

O 'Jogo da Memória: conhecendo os sintomas da COVID-19' foi proposto para abordar desde os sintomas leves até os mais graves ligados à doença, a fim de promover a familiarização e reconhecimento desses sintomas caso necessário. A proposta do jogo interativo 'Como Controlar o Coronavírus' foi proporcionar informações sobre as medidas de distanciamento e isolamento social por meio de estratégias de associação.

O intuito da 'Cruzadinha da COVID-19' foi utilizar de forma criativa palavras-chave relacionadas à prevenção da doença. Abordagens ligadas à manutenção da saúde mental dos idosos foram utilizadas no 'Bingo da COVID-19' por meio de frases de conforto.

Já o folder 'Coronavírus e a COVID-19: Você sabia?' propôs uma maneira simples e resumida para diferenciar os tipos de coronavírus e ressaltar a inexistência da transmissão do SARS-CoV-2 de animais para humanos.

\section{Material didático digital}

Todo material digital produzido foi disponibilizado às instituições via e-maile em CD gravado para cada abrigo de idosos. Foram elaborados dois vídeos informativos, um jogo digital e uma história em quadrinhos. Os vídeos foram denominados 'Que vírus é esse?' e 'Queridos idosos', o jogo interativo de 'Verdades e Mitos da Prevenção da COVID-19' e a história em quadrinhos de 'Diálogo entre o Coronavírus x Mundo'.

O objetivo do vídeo 'Que vírus é esse?' foi esclarecer as características gerais do vírus de maneira simples e ilustrativa. Já no vídeo 'Queridos idosos' a ideia foi esquematizar medidas de controle aliadas a mensagens de carinho e conforto. O jogo 'Verdades e Mitos da Prevenção da COVID-19' enfatizou o entendimento sobre a doença por meio de perguntas sobre as formas de transmissão e prevenção. A proposta da história em quadrinhos 'Coronavírus x Mundo', por sua vez, foi informar sobre a origem e as 'predileções' do SARS-CoV-2 (ou seja, situações que o favorecem), de maneira lúdica, com linguagem informal.

\section{Aplicação prática}

A necessidade de respeitar as medidas de distanciamento social fez com que a ideia inicial do projeto fosse adaptada à aplicação dos jogos pelos próprios funcionários e assistentes sociais de cada abrigo. Por isso, em todas as propostas foram desenvolvidas regras específicas de aplicação explicadas aos funcionários nos próprios jogos, via videoconferência, contato telefônico e/ou e-mail.

Todas as entregas foram realizadas pessoalmente, respeitando as medidas de distanciamento social. Os materiais didáticos foram apresentados aos idosos em nove dias distintos, de acordo com o cronograma de atividades local, que seguiu a ordem estabelecida na Tabela 1, para garantir a aquisição gradativa do conhecimento. Todas as aplicações práticas foram acompanhadas via videoconferência, gravações e/ou fotos. 
Tabela 1. Ordem estabelecida de aplicação das atividades propostas aos idosos para o processo ensinoaprendizagem gradual sobre a COVID-19.

\begin{tabular}{|c|c|c|}
\hline Ordem & Atividade & Justificativa \\
\hline $1^{\circ} \mathrm{dia}$ & $\begin{array}{l}\text { Vídeo 'Que vírus é esse' e contato com o vírus } \\
\text { de biscuit }\end{array}$ & Conhecimento inicial sobre o agente. \\
\hline $2^{\circ} \mathrm{dia}$ & Folder 'Coronavírus e a COVID-19: Você sabia?' & $\begin{array}{l}\text { Esclarecimento sobre origem e a relação } \\
\text { com animais. }\end{array}$ \\
\hline $3^{\circ} \mathrm{dia}$ & Jogo digital 'Verdades e Mitos da COVID-19' & $\begin{array}{l}\text { Abordagem principal sobre os aspectos } \\
\text { ligados à transmissão da doença. }\end{array}$ \\
\hline $4^{\circ} \mathrm{dia}$ & $\begin{array}{l}\text { 'Jogo da memória: conhecendo os sintomas da } \\
\text { COVID-19' }\end{array}$ & $\begin{array}{l}\text { Compreensão dos diferentes sinais } \\
\text { clínicos e dos níveis de gravidade. }\end{array}$ \\
\hline $5^{\circ} \mathrm{dia}$ & 'Cruzadinha da COVID-19' & \multirow{3}{*}{$\begin{array}{l}\text { Aprofundamento e fixação acerca das } \\
\text { medidas de controle e prevenção da } \\
\text { COVID-19. }\end{array}$} \\
\hline $6^{\circ}$ dia & Jogo 'Como Controlar o Coronavírus' & \\
\hline $7^{\circ} \mathrm{dia}$ & $\begin{array}{l}\text { História em quadrinhos digital 'Coronavírus x } \\
\text { Mundo' }\end{array}$ & \\
\hline $8^{\circ} \mathrm{dia}$ & 'Bingo da COVID-19' & \multirow{2}{*}{$\begin{array}{l}\text { Apoio às questões relativas à saúde } \\
\text { mental. }\end{array}$} \\
\hline $9^{\circ} \mathrm{dia}$ & Vídeo 'Queridos Idosos' & \\
\hline
\end{tabular}

\section{Resultados e Discussão}

O trabalho foi realizado a partir de dez atividades educativas, a fim de levar informações completas sobre a COVID-19 aos idosos residentes de 16 casas de repouso da cidade de Uberlândia, Minas Gerais. As atividades foram realizadas do mês de abril ao mês de junho de 2020. Ao todo, foram 364 residentes beneficiados com os materiais didáticos, além de toda a equipe de funcionários em contato direto com os idosos. De maneira complementar, foram arrecadados e doados, a partir de empresas e de pessoas físicas, produtos de limpeza, de higiene pessoal, máscaras e gêneros alimentícios, com o intuito de enfatizar as ações práticas voltadas à prevenção dessa doença.

Quanto às atividades educativas, os jogos interativos propostos foram 'Verdades e mitos da prevenção da COVID-19' (Figura 1a), 'Bingo da COVID-19', 'Cruzadinha da COVID-19', 'Como Controlar o Coronavírus' e 'Jogo da Memória: conhecendo os sintomas da COVID-19', todos acompanhados com as devidas instruções para auxiliar o mediador no momento da aplicação. O material informativo produzido consistiu dos vídeos 'Que vírus é esse?' - acompanhado do vírus de biscuit para facilitar a compreensão - e 'Queridos idosos' (Figura 1b), além do folder: 'Coronavírus e a COVID-19: Você sabia?' e da história em quadrinhos 'Diálogo entre o Coronavírus x Mundo'. Os materiais distribuídos foram aplicados pelas equipes de assistência social e terapia ocupacional de cada asilo (Figura 2), uma vez que as medidas de isolamento e distanciamento social 
impossibilitaram nossa participação direta.
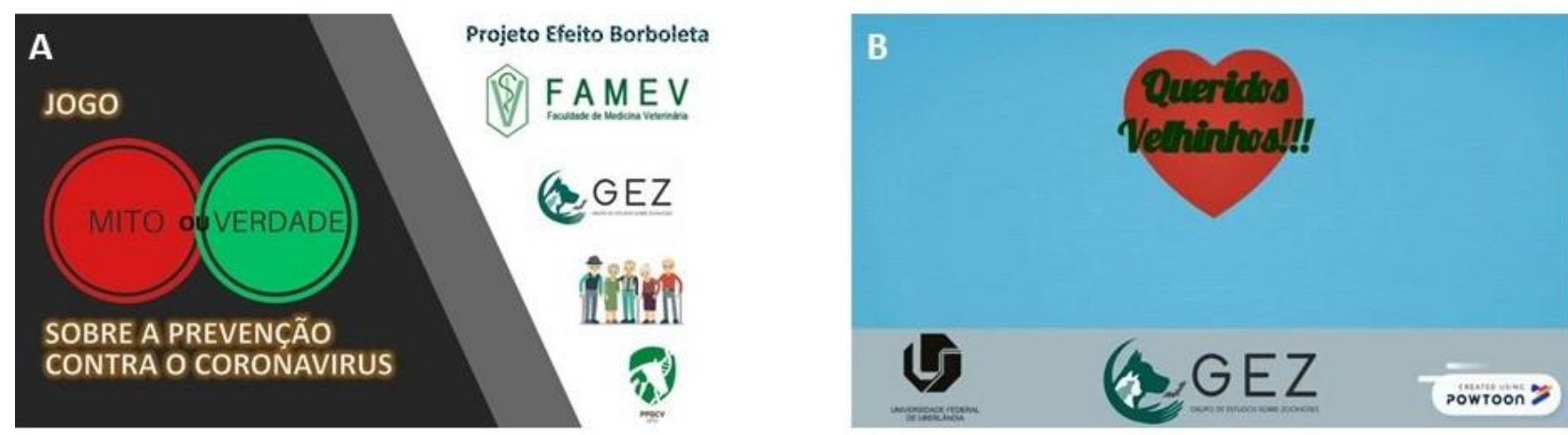

Figura 1. Materiais educativos desenvolvidos nos formatos físico e digital - 'Verdades e mitos da prevenção da COVID-1.9' (A) e vídeo 'Queridos idosos' (B).
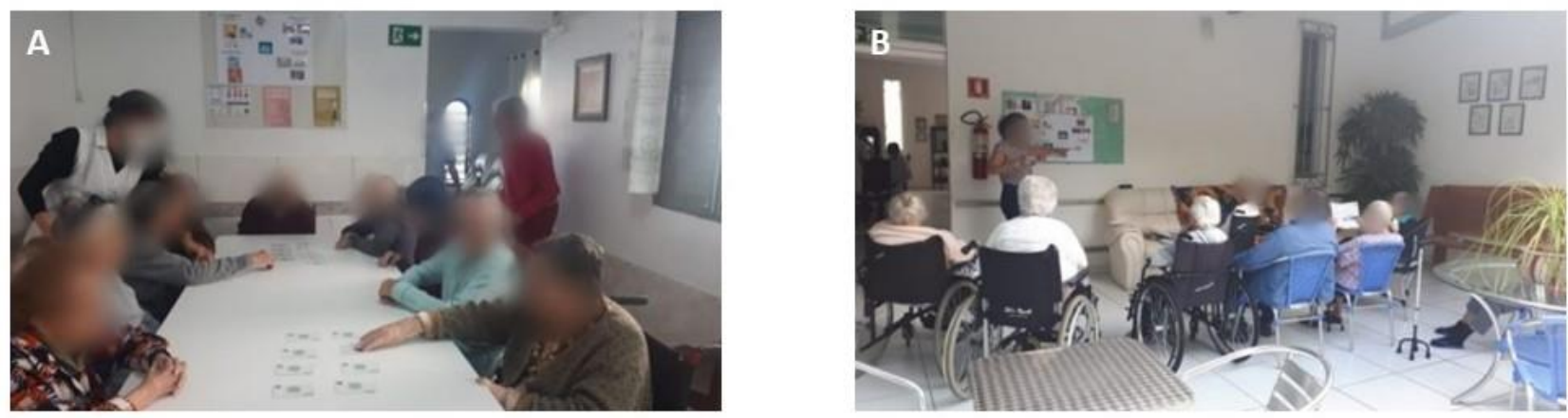

Figura 2. Momento de aplicação dos materiais didáticos: 'Jogo da Memória: conhecendo os sintomas da COVID-19' (A) e jogo 'Jogo Como Controlar o Coronavírus' (B), nos diferentes asilos beneficiados.

Antes da realização das entregas dos materiais didáticos e das doações aos asilos, nos foi relatado que grande parte dos idosos apresentava elevada resistência em seguir as recomendações de combate à doença, em relação ao contato físico e isolamento social, principalmente. No Brasil, culturalmente, há maior proximidade física entre a população, de modo que a transmissão da doença é favorecida, e os meios de comunicação que retratam as medidas de prevenção e controle são de difícil compreensão, especialmente entre as pessoas de idade avançada.

A partir dos resultados e relatos feitos posteriormente pela equipe de enfermeiros, terapeutas ocupacionais e assistentes sociais de cada casa de repouso, os materiais didáticos permitiram uma maior conscientização e adesão por parte dos idosos em relação às medidas de controle e prevenção da COVID-19. De acordo com Damasceno et al. (2018), ações educativas são fundamentais no envolvimento dos idosos quanto ao cuidado com sua saúde. Além disso, proporcionam maior interação entre os profissionais, familiares e idosos, o que possibilita a troca de informações e favorece hábitos mais saudáveis, importantes no controle de diversas doenças e complicações. Logo, a conscientização através de medidas educativas lúdicas sobre a COVID-19 é importante para essa faixa-etária, de modo a envolver ativamente os idosos no cuidado com a saúde física e mental nas casas de repouso, além do impacto positivo a toda comunidade de convivência com estes idosos.

O vídeo 'Que Vírus é Esse?' representou uma forma de interação digital para divulgação do conhecimento e consequente promoção da saúde. O material potencializou o alcance da informação por meio do 
esclarecimento de dúvidas sobre características e funcionalidades do vírus causador da doença. Essa compreensão deixa claros os fundamentos das formas de prevenção e facilita a sua aceitação e adaptação pelos idosos. O 'vírus de biscuit' complementou de forma palpável essas características e induziu reflexões e discussões que favorecem a fixação do conteúdo.

O folder 'Coronavírus e a COVID-19: Você Sabia?' consistiu em informações sobre a origem da doença e as diferenças do SARS-CoV-2 com os demais coronavírus que afetam animais. O material informativo contemplou imagens de animais com a descrição simples dos outros coronavírus, focando no aprendizado visual. A ideia central foi deixar claro que o contato com animais não favorece a transmissão da COVID-19. Até o momento, sabe-se que o SARS-CoV-2 provavelmente apresentou como hospedeiro primário os morcegos, que abrigam uma variedade de subtipos de coronavírus, de maneira comensal. Por meio de transferência adaptativa, esse vírus sofreu processos de recombinação, tendo os pangolins como hospedeiros intermediários e humanos como hospedeiros finais. De maneira concomitante, vários estudos têm tentado mostrar o risco de transmissão da COVID-19 a partir de animais, como furões, gatos, cães, porcos, galinhas e patos, para humanos (Shi et al., 2020; Zhang et al., 2020), mas nenhum comprova seu risco efetivo, e são questionados quanto ao desenho experimental (Hora, 2020).

O material digital 'Verdades e Mitos da Prevenção da COVID-19' permitiu momentos de descontração, ao mesmo tempo em que testava o conhecimento dos envolvidos. Consistiu em 11 perguntas que o jogador deveria responder quanto à veracidade, sendo vencedor aquele que acertasse mais de sete questões. $A$ abordagem focou nas formas de transmissão da COVID-19. De acordo com o Ministério da Saúde (2020b), a principal forma de disseminar a doença se dá por contato direto entre as pessoas, por meio de aperto de mãos, gotículas de saliva, espirro, tosse, secreções nasais e objetos ou superfícies contaminadas, como mesas, maçanetas, entre outros itens compartilhados, seguido do contato do vírus com as mucosas ocular, nasal e bucal do paciente.

Outro material físico foi o 'Jogo da Memória: conhecendo os sintomas da COVID-19', que abordou aspectos ligados aos sintomas da COVID-19, subdivididos em três categorias, conforme a gravidade. A atividade apresentou 16 pares de figuras correspondentes ao nome do sintoma, acompanhados das instruções do jogo e de um informativo que discrimina os sintomas no humano conforme a intensidade. Os sintomas foram divididos em respiratórios, digestivos e sistêmicos, e incluíram: dor de cabeça, dor nas articulações, tosse seca, febre, dificuldade de respirar, pneumonia, falência renal, diarreia, perda do olfato, insuficiência respiratória, dor abdominal, náusea, coriza, dor muscular, dor de garganta e vômito. Sabe-se que a COVID-19 está associada especialmente a alterações respiratórias, e que a maioria das pessoas infectadas apresenta sintomas leves e se recupera. Em alguns casos os sintomas nem sequer são apresentados. Porém, a doença pode levar também a sintomas mais graves, como a pneumonia, e, dependendo do grau de debilidade, à morte, mais relevante em pacientes idosos (Huang et al., 2020).

A 'Cruzadinha da COVID-19' possibilitou o aprendizado individual por meio de palavras cruzadas com informações sobre o controle e a prevenção da doença, utilizando uma linguagem acessível. Sua composição incluía 14 palavras-chave dispostas vertical e horizontalmente, cruzando-se umas com as outras, além de dicas enumeradas sobre cada palavra a ser descoberta, estimulando o aprendizado.

De maneira semelhante, o jogo 'Como Controlar o Coronavírus' permitiu a disseminação de informações ligadas ao combate à pandemia de maneira coletiva e interativa. A atividade era composta por um cartão de definições, um cartão de respostas, um quadro produzido em cartolina, o qual possuía quatro áreas delimitadas com as expressões 'Isolamento social', 'Distanciamento social', 'Quarentena' e 'Bloqueio total', além de 12 imagens, as quais o jogador teria que distribuir conforme os diferentes conceitos. $O$ vencedor seria $O$ jogador/grupo que mais acertasse as figuras colocadas nas áreas adequadas. 
Seguindo a mesma ideia de controle da COVID-19, foi desenvolvido ainda um diálogo digital denominado 'Coronavírus x Mundo', no qual o coronavírus conversa com o mundo, de maneira lúdica, sobre seu surgimento e sobre os cuidados que o mundo deve tomar que impedem sua propagação. O material foi elaborado seguindo a ideia de história em quadrinhos, unindo texto e imagens ao narrar o diálogo, de fácil entendimento ao leitor (disponível em Material Suplementar 2).

A maior quantidade de materiais produzidos focou nas medidas de controle e prevenção da doença pela necessidade imperativa de levar esse tipo de informação e sensibilizar ao máximo os idosos a essas novas regras. Devido à rápida disseminação da COVID-19 e ao alto poder de contágio do vírus, além da ausência de vacinas e antivirais, as autoridades de saúde têm buscado implementar medidas tradicionais de Saúde Pública para conter a doença (Wilder-Smith \& Freedman, 2020). Essas medidas consistem na redução da movimentação e do contato entre as pessoas, dada a maneira de transmissão do vírus, além das formas básicas de higiene, como lavar as mãos e higienizar adequadamente as superfícies. A conscientização sobre essas medidas, aliada à distribuição de produtos aplicáveis a essas necessidades e à atual realidade, representou nossa principal estratégia para efetivar a promoção da saúde dos envolvidos.

O 'Bingo da COVID-19', por sua vez, utilizou de palavras de encorajamento ao público-alvo do projeto. O jogo consistiu em uma adaptação do bingo tradicional, no qual, em vez de bolas enumeradas, havia 50 palavras/frases motivadoras dispostas aleatoriamente em 20 cartelas compostas de cinco linhas e cinco colunas. A ideia central do jogo era favorecer a saúde mental, que se encontra mais debilitada nesse momento de pandemia. Paralelamente, o vídeo 'Queridos Idosos', apresentou de maneira digital uma homenagem aos idosos, que valorizou a importância referencial do grupo à sociedade e reforçou a ideia de exemplos de vida aos seus familiares. Se, por um lado, há necessidade de inclusão de cuidados voltados às restrições e medidas de isolamento social, por outro, é também essencial o atendimento centrado na pessoa idosa e no cuidado à manutenção da qualidade de vida, reforçando a importância dessa classe social, mediada pela interação carinhosa com os residentes, apoiados na atenção psicossocial (Wang et al., 2020).

Quanto aos resultados quantitativos observados, dos 16 asilos beneficiados pelo projeto, obtivemos o retorno de 14 das instituições quanto aos casos de COVID-19. Felizmente, até o encerramento das ações de extensão (junho de 2020), não houve nenhum caso positivo da doença nos idosos desses locais. Além disso, os idosos demonstraram maior sensibilização à adoção dos cuidados contra o coronavírus. Tais fatos indicam o impacto positivo do projeto no controle e prevenção da doença ao público-alvo, e corrobora com o que foi observado por Damasceno et al. (2018), em estudo sobre a Extensão Universitária como estratégia para a Educação em Saúde com um grupo de idosos. Os autores observaram que a inserção de ações universitárias junto aos idosos permitiram o desenvolvimento de uma abordagem holística e aprendizagem significativa, alcançando a promoção da saúde, recuperação e, principalmente, a prevenção de doenças, atendendo às necessidades reais do público-alvo assistido.

Para reforçar as ações, foram realizadas doações às instituições envolvidas, por meio de parcerias com indivíduos e empresas de diversos segmentos, como clínicas veterinárias, consultórios de psicologia, serviços de alimentação, fábricas de produtos de limpeza e higiene pessoal, drogarias e farmácias de manipulação veterinária. Os insumos adquiridos incluíram gêneros alimentícios, itens de limpeza e higiene pessoal, além de máscaras, tanto para os idosos quanto para seus colaboradores. Os materiais arrecadados contribuíram para a aplicação de forma prática do conteúdo abordado, principalmente o referente ao controle e prevenção da doença. De acordo com a World Health Organization (WHO, 2020), a recepção de doações gera entusiasmo nas pessoas envolvidas, e se estabelece como importante estratégia de superação ou como um fator de sucesso no enfrentamento de agravos, como a COVID-19. A abordagem positiva sobre as doações se alinha às estratégias de acesso facilitado às formas de prevenção da pandemia. Dessa maneira, o impacto dessa ação tende a gerar uma motivação na comunidade geral no combate à COVID-19, no que se refere ao cuidado 
próprio e dos funcionários com os idosos.

Algumas dificuldades foram encontradas na realização do projeto, sendo principalmente relacionadas aos recursos necessários para a confecção das atividades propostas, que foram supridas por meio de doações provenientes de cinco empresas do ramo de papelaria. As restrições relacionadas à execução à distância das atividades, por sua vez, culminaram em atrasos na produção dos materiais e no estabelecimento de contatos entre os asilos, as empresas parceiras e a comunidade. Além disso, as restrições de contato físico e as medidas de isolamento social limitaram as etapas de entrega e realização das atividades práticas junto aos idosos por parte do grupo. No entanto, tais adversidades foram contornadas por meio de ajustes no cronograma de entregas e contatos estabelecidos, para a sincronia das atividades. De maneira complementar, foram incluídas instruções específicas a cada material, aliado a uma comunicação reafirmada periodicamente junto às casas de repouso, a fim de esclarecer aos funcionários o detalhamento sobre as atividades a serem realizadas com os idosos.

De maneira geral, as atividades permitiram reflexões sobre prevenção da COVID-19 e promoção de saúde, tanto nos idosos, quanto na equipe de seu convívio. O material didático contribuiu no incentivo aos idosos a seguirem as recomendações das autoridades de saúde, ao transmitir, de maneira lúdica e informal, a importância dessas ações, bem como a maneira de executá-las.

\section{Considerações finais}

A extensão universitária favoreceu a execução de ações na comunidade de forma simultânea à construção do conhecimento, unindo tanto o saber acadêmico quanto a linguagem lúdica, para intensificar os vínculos e permitir o empoderamento tanto dos estudantes, quanto dos profissionais e idosos de cada instituição participante. Foi perceptível o sentimento de gratidão de todas as partes envolvidas no projeto. Além disso, foi visível o impacto positivo para o controle e a prevenção da doença, uma vez que houve estímulo a um maior engajamento por parte da comunidade em relação ao cuidado com os idosos e valorização desses indivíduos, especialmente neste período de pandemia.

\section{Agradecimentos}

Às empresas e pessoas envolvidas nas doações.

\section{Contribuição de cada autor}

A.L.M.F. coordenou a arrecadação das doações, participou das entregas e contribuiu na introdução; R.F.B. estabeleceu os contatos, participou das entregas, contribuiu na discussão e adequou o inglês e as normas; M.C.C. coordenou os materiais físicos, participou das entregas e contribuiu nos resultados; D.A.P. elaborou jogos digitais, contribuiu na arrecadação de doações, na divulgação e nas considerações finais; A.B.S. e D.R.P. elaboraram materiais físicos e digitais e contribuíram nos procedimentos metodológicos. R.T.M coordenou e orientou todas as etapas.

\section{Referências}

Alzheimer Europe. (2020). Alzheimer Europe recommendations on promoting the wellbeing of people with dementia and carers during the COVID-19 pandemic. Luxemburg: Alzheimer Europe. Recuperado de https://www.alzheimereurope.org/Policy/Our-opinion-on/Wellbeing-of-people-with-dementia-during-COVID-19-pandemic 
Brown, C. (2003). Virchow revisited: Emerging zoonoses. American Society for Microbiology, 69(10), 493-497.

Chen, Y., Liu, Q., \& Guo, D. (2020). Emerging coronaviruses: Genome structure, replication, and pathogenesis. Journal of Medical Virology, 92(4), 418-423.

Damasceno, A. J. S., Aragão, C. P., Mesquita, F. M. S., Vasconcelos, V. P., de Sousa, L. S., de Sousa, L. V., \& Moreira, A. C. A. (2018). A Extensão Universitária como estratégia para a Educação em Saúde com um grupo de idosos. Revista Kairós: Gerontologia, 21(4), 317-333.

Hora, A. S. (2020). Coronavirus: A veterinary perspective. Nature, 580(7803), 321-321.

Huang, X., Wei, F., Hu, L., Wen, L., \& Chen, K. (2020). Epidemiology and clinical characteristics of COVID-19. Archives of Iranian Medicine, 23(4), 268-271.

King, L. J., Anderson, L. R., Blackmore, C. G., Blackwell, M. J., Lautner, E. A., Marcus, L. C., ... \& Mahr, R. K. (2008). Executive summary of the AVMA One Health Initiative Task Force report. Journal of the American Veterinary Medical Association, 233(2), 259-261.

McMichael, T. M., Currie, D. W., Clark, S., Pogosjans, S., Kay, M., Schwartz, N. G., ... \& Duchin, J. S. (2020). Epidemiology of Covid-19 in a Long-Term Care Facility in King Country, Washington. The New England Journal of Medicine, 382(21), 2005-2011.

Ministério da Saúde. (2020a). Guia de Vigilância Epidemiológica do COVID-19. Brasília: Ministério da Saúde do Brasil. Recuperado de https://covid.saude.gov.br/

Ministério da Saúde. (2020b). Coronavírus (COVID-19). Brasília: Ministério da Saúde do Brasil. Recuperado de https://coronavirus.saude.gov.br/

Read, S., Comas-Herrera, A., \& Grundy, E. (2020). Social isolation and memory decline in later-life. The Journals of Gerontology: Series B, 75(2), 367-376.

Shi, J., Wen, Z., Zhong, G., Yang, H., Wang, C., Huang, B., ... \& Zhao, Y. (2020). Susceptibility of ferrets, cats, dogs, and other domesticated animals to SARS-coronavirus 2. Science, 368(6494), 1016-1020.

Wang, H., Li, T., Barbarino, P., Gauthier, S., Brodaty, H., Molinuevo, J. L., ... \& Weidner, W. (2020). Dementia care during COVID-19. The Lancet, 395(10231), 1190-1191.

Wilder-Smith, A., \& Freedman, D. O. (2020). Isolation, quarantine, social distancing and community containment: pivotal role for old-style public health measures in the novel coronavirus (2019-nCoV) outbreak. Journal of Travel Medicine, $27(2), 1-4$.

World Health Organization. (2020). Infection Prevention and Control guidance for Long-Term Care Facilities in the context of COVID-19. [ S. I. ]: WHO. Recuperado de https://apps.who.int/iris/bitstream/handle/10665/331508/WHO-2019nCoV-IPC long term care-2020.1-eng.pdf?sequence=1\&isAllowed=y

Zhang, T., Wu, Q., \& Zhang, Z. (2020). Probable pangolin origin of SARS-CoV-2 associated with the COVID-19 outbreak. Current Biology, 30(8), 1346-1351.

\section{Como citar este artigo:}

Ferreira, A. L. M., Braz, R. F., Chueiri, M. C., Policarpo, D. A., Siqueira, A. B., Pacheco, D. R., Melo, R. T. (2020). Medicina veterinária e a saúde única: Ação socioeducativa aplicada a idosos no contexto da COVID-19. Revista Brasileira de Extensão Universitária, 11(3), 429-438. https://periodicos.uffs.edu.br/index.php/RBEU/article/view/11560/pdf 